\title{
Adaptation of a Styrene-Acrylic Acid Copolymer Surface to Water
}

\author{
Xiaomei Li, Simon Silge, Alexander Saal, Gunnar Kircher, Kaloian Koynov, Rüdiger Berger,* \\ and Hans-Jürgen Butt
}

Cite This: Langmuir 2021, 37, 1571-1577

Read Online

\section{ACCESS | Lلll Metrics \& More | 国 Article Recommendations | Sl Supporting Information}

ABSTRACT: Solid surfaces, in particular polymer surfaces, are able to adapt upon contact with a liquid. Adaptation results in an increase in contact angle hysteresis and influences the mobility of sliding drops on surfaces. To study adaptation and its kinetics, we synthesized a random copolymer composed of styrene and 11-25 mol\% acrylic acid (PS/PAA). We measured the dynamic advancing $\left(\theta_{\mathrm{A}}\right)$ and receding $\left(\theta_{\mathrm{R}}\right)$ contact angles of water drops sliding down a tilted plate coated with this polymer. We measured $\theta_{\mathrm{A}} \approx 87^{\circ}$ for velocities of the contact line $<20 \mu \mathrm{m} / \mathrm{s}$. At higher velocities, $\theta_{\mathrm{A}}$ gradually increased to $\sim 98^{\circ}$. This value is similar to $\theta_{\mathrm{A}}$ of a pure polystyrene (PS) film, which we studied for comparison. We associate the gradual increase in $\theta_{\mathrm{A}}$ to the adaptation process to

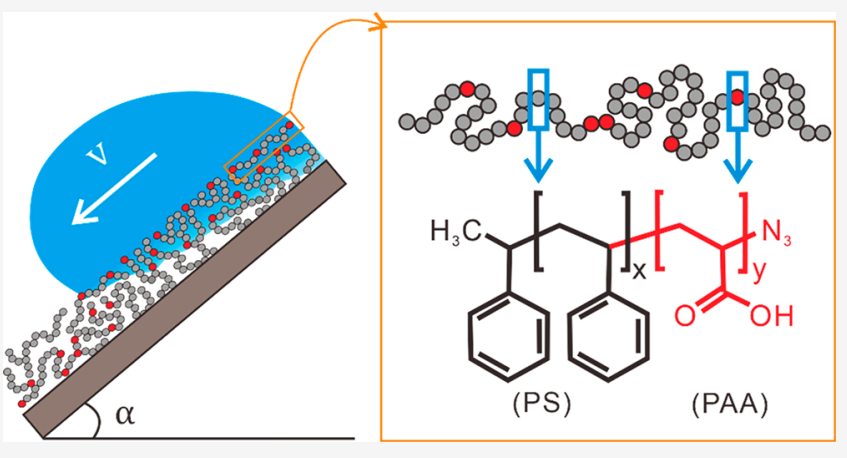
water: The presence of water leads to swelling and/or an enrichment of acid groups at the water/polymer interface. By applying the latest adaptation theory (Butt et al. Langmuir 2018, 34, 11292), we estimated the time constant of this adaptation process to be $\ll 1$ s. For sliding water drops, $\theta_{\mathrm{R}}$ is $\sim 10^{\circ}$ lower compared to the reference PS surface for all tested velocities. Thus, at the receding side of a sliding drop, the surface is already enriched by acid groups. For a water drop with a width of $5 \mathrm{~mm}$, the increase in contact angle hysteresis corresponds to an increase in capillary force in the range of $45-60 \mu \mathrm{N}$, depending on sliding velocity.

\section{INTRODUCTION}

Many surfaces react when they are brought into contact with a liquid. Some polymer surfaces, for example, reconstruct due to a reorientation of side groups or due to selective exposure of specific segments to the liquid interface. ${ }^{1-4}$ In addition, polymers swell when liquid diffuses into the polymer. ${ }^{5-7}$ Mixed polymer brushes, or block copolymers, expose the more compatible component to the liquid interface. ${ }^{8-10}$ Adaptation of the surface typically leads to changes in surface composition upon exposure to a liquid. Consequently, adaptation contributes to contact angle hysteresis and dynamic contact angle changes. ${ }^{11}$ These parameters are important in the fields of printing, digital microfluidics, and fiber coatings. Despite the importance in many wetting applications, a quantitative understanding of adaptation is still in its infancy.

When adaptation is nonreversible upon exposure to air, the adaptive kinetics at the solid/liquid interface can be probed by immersing the sample in liquids for different time spans. Subsequently, the surface can be studied by X-ray photoelectron spectroscopy (XPS). ${ }^{12-17}$ However, reversible adaptation cannot be studied by XPS as it requires vacuum conditions. In addition, studying adaptation kinetics with a time scale of $<1 \mathrm{~s}$ is challenging due to the time required to immerse samples into and remove samples from the liquid. Thus, measurements locoated directly at, or close to, the moving three-phase contact line are beneficial.
Contact angle measurements are an elegant way of measuring adaptation kinetics. ${ }^{18-20}$ Surface adaptation leads to a change in advancing and receding contact angles, ${ }^{11}$ which is experimentally easily measurable. Surface adaptation kinetics have been probed by measuring time-dependent dynamic contact angles using the Wilhelmy method, or by using the infused drop method. ${ }^{21-24}$ Both methods can reveal changes in contact angles caused by surface adaptation. However, the studied adaptation processes are rather slow; they range in time from several minutes to several days. ${ }^{22,25-27}$ To our knowledge, no report of direct contact angle measurements exists that reveals adaptation velocities of surfaces faster than seconds.

A quantitative description of the adaptive wetting phenomena was recently outlined by Butt et al. They introduced exponentially relaxing interfacial energies and applied Young's equation locally. The theory predicts the advancing $\left(\theta_{\mathrm{A}}\right)$ and receding $\left(\theta_{\mathrm{R}}\right)$ contact angles of a sliding drop on a surface exhibiting specific adaptation kinetics: ${ }^{11}$

Received: November 8, 2020

Revised: December 24, 2020

Published: January 13, 2021 


$$
\begin{aligned}
& \cos \theta_{\mathrm{A}}=\cos \theta^{\infty}-\frac{\Delta \gamma_{\mathrm{SL}}}{\gamma_{\mathrm{L}}^{\infty}} \mathrm{e}^{-l_{\mathrm{SL}} / v \tau_{\mathrm{SL}}} \\
& \cos \theta_{\mathrm{R}}=\cos \theta^{\infty}+\frac{\Delta \gamma_{\mathrm{S}}}{\gamma_{\mathrm{L}}^{\infty}} \mathrm{e}^{-l_{\mathrm{S}} / v \tau_{\mathrm{S}}}
\end{aligned}
$$

$\theta^{\infty}$ is the contact angle in thermodynamic equilibrium (that is, for velocity $v \rightarrow 0$ ), and $\gamma_{\mathrm{L}}^{\infty}$ is the liquid surface tension. $\Delta \gamma_{\mathrm{SL}}$ and $\Delta \gamma_{S}$ quantify the change in solid/liquid interfacial energy due to adaptation and the change in solid surface energy due to adaptation after the surface becomes dry, respectively. $\tau_{\mathrm{SL}}$ and $\tau_{\mathrm{S}}$ are relaxation times for the adaptation processes of the solid surface after it comes into contact with the liquid and dries again. $l_{\mathrm{SL}}$ and $l_{\mathrm{S}}$ are peripheral thicknesses, which are the spans of the contact region that influence the advancing and receding contact angles, respectively. According to this theory, contact angle measurements allow us to calculate the ratio of the peripheral length and the relaxation time. The kinetics, i.e., relaxation time of adaptation can then be anticipated by assuming a peripheral length value. To date, experimental verification of this adaptation theory is still lacking.

To explore the adaptation kinetics of surfaces upon contact with a liquid at a time scale $<1 \mathrm{~s}$, we designed and built a tiltedplate setup allowing us to measure velocity-dependent dynamic contact angles. As an adaptive surface, we used films of a random copolymer consisting of hydrophobic styrene (majority) and hydrophilic acrylic acid (minority) monomers. We studied the adaptation of these films to sliding water drops.

\section{METHODS}

Substrate Cleaning. Si wafers and cover glass slides were cleaned by ultrasonication in toluene (98\%, Sigma), ethanol (99.5\%, Sigma), and deionized (DI) water for $10 \mathrm{~min}$, respectively. Then the substrates were dried using a nitrogen stream. Before coating, the substrates were further cleaned or activated for $10 \mathrm{~min}$ in a gas plasma made by $100 \%$ oxygen.

Preparation of the PS/PAA Surface. To synthesize PS/PAA copolymers, $22 \mathrm{~mL}$ of styrene, $2 \mathrm{~mL}$ of acrylic acid, and $60 \mathrm{mg}$ of azobis(isobutyronitrile) (98\%, Sigma) were dissolved in $30 \mathrm{~mL}$ of dimethylformamide (DMF) (99.8\%, Sigma). The solution was degassed by three freeze/pump/thaw cycles and then stirred at $70{ }^{\circ} \mathrm{C}$ for $48 \mathrm{~h}$. After it was precipitated three times using methanol, we obtained the copolymer.

To fabricate a PS/PAA film, $0.5-1$ wt \% PS/PAA copolymer in tetrahydrofuran (THF) (99.9\%, Sigma) solution was dropped onto the Si wafer. Spinning was performed for $60 \mathrm{~s}$ at $1000 \mathrm{rpm}$. Then, the samples were heated at $150{ }^{\circ} \mathrm{C}$ in a vacuum overnight. Films with thicknesses between 30 and $70 \mathrm{~nm}$ were obtained.

Preparation of the PS Surface. PS was synthesized by anionic polymerization. The glassware was baked under vacuum, and then $150 \mathrm{~mL}$ of cyclohexane $(99.8 \%$, Sigma) with dissolved starter ( secbutyllithium) was added. Then $20 \mathrm{~g}$ of styrene was added. The mixture reacted overnight at room temperature. The polymerization was stopped by adding a few $\mathrm{mL}$ of degassed methanol. Subsequently more degassed methanol was added until the PS precipitated. The precipitate was dissolved in THF again and reprecipitated with degassed methanol.

To coat a Si wafer with PS, 2 wt \% PS in toluene was dropped onto the $\mathrm{Si}$ wafer and then spinning was performed for $60 \mathrm{~s}$ at $2000 \mathrm{rpm}$. Finally, the samples were heated at $120{ }^{\circ} \mathrm{C}$ in vacuum overnight.

Preparation of the PS/PAA Surface with pH-Sensitive Dye. After cleaning and activation, cover glass slides were immersed in $2 \mathrm{wt}$ \% (3-aminopropyl)triethoxysilane (APTES, 98\%, Sigma) anhydrous toluene (99.8\%, Sigma) solutions for $4 \mathrm{~h}$ to form an APTES monolayer as a precursor. The slides were then sonicoated twice in toluene for 10 min to remove physisorbed APTES. After drying in a stream of nitrogen gas, the pH-sensitive dye (pHrodo iFL STP EATER, ThermoFisher) was grafted onto the APTES slides by immersing them in a mixture of $0.1 \mathrm{M}$ sodium bicarbonate buffer $(\mathrm{pH}$ 8.2 ) and 5 wt \% dimethyl sulfoxide (99.7\%, Sigma) at a dye concentration of $100 \mu \mathrm{g} / \mathrm{mL}$ for $3 \mathrm{~h}$. Afterward, the slides were sonicated twice in deionized water for $10 \mathrm{~min}$ and dried with nitrogen gas. Finally, the 8.7/1 PS/PAA copolymer was spin-coated onto the slides with a dye layer using $0.8 \mathrm{wt} \%$ THF solution and a spinning speed of $1000 \mathrm{rpm}$ for $60 \mathrm{~s}$.

Contact Angle Measurement Using the Sessile Drop Method. Contact angles were measured in the sessile drop configuration (Dataphysics contact angle measurement system, OCA35). An $8 \mu \mathrm{L}$ water drop was deposited on the surface. Then $16 \mu \mathrm{L}$ of DI water was pumped into the drop by a Hamilton syringe $(100 \mu \mathrm{L})$ with a hydrophobic needle. Then $16 \mu \mathrm{L}$ of water was sucked out of the drop (flow rate $=0.5 \mu \mathrm{L} / \mathrm{s}$, mean contact line velocity $=(8$ $\left.\pm 0.5) \times 10^{-5} \mathrm{~m} / \mathrm{s}\right)$. The process was repeated five times without interruption. Inflation and deflation were imaged using a high-speed camera taking side images. The advancing and receding contact angles were calculated by fitting an elliptical model to the images recorded in side view.

Dynamic Contact Angle Measurement Using the TiltedPlate Setup. A $33 \pm 1 \mu \mathrm{L}$ water drop was pipetted onto the tilted surface using a syringe pump (KD Scientific, Legato 100 Syringe Pump). The tilt angles of the surface were varied from $33^{\circ}$ to $70^{\circ}$ for an $8.7 / 1$ PS/PAA surface to adjust the drop velocity. In addition, different sliding velocities of drops were accomplished by measuring the drop at different distances from the position where the drop was released. The interval between drops was $10 \mathrm{~min}$ for PS surfaces. PS/ PAA surfaces were annealed to $150{ }^{\circ} \mathrm{C}$ for $10 \mathrm{~min}$ between measurements.

To check if the interval between drops was long enough to dry the surface, a series of 40 drops with a volume of $33 \pm 1 \mu \mathrm{L}$ was pipetted onto the $67 \mathrm{~nm} 8.7 / 1 \mathrm{PS} / \mathrm{PAA}$ surface and the PS surface using the syringe pump. We set the interval between individual drops to $1 \mathrm{~min}$ for PS surfaces. For the PS/PAA surface, we set it to $10 \mathrm{~min}$ as it includes a heating step at $150{ }^{\circ} \mathrm{C}$. The inclination angle was kept constant: $\alpha=30^{\circ}$ for the PS/PAA surface, and $\alpha=16^{\circ}$ for the PS surface. The distance between the pipet and the camera's field of view ranges from 3 to $6 \mathrm{~cm}$. To prevent the results being affected by surface inhomogeneity, the contact angle was always measured at the same spot on the samples.

Confocal Laser Scanning Microscopy Measurement. Confocal laser scanning microscopy (CLSM) experiments were performed on a commercial confocal microscope, LSM 510 (Carl Zeiss, Jena, Germany) equipped with a C-Apochromat 40/1.2 W waterimmersion objective. For excitation, the $488 \mathrm{~nm}$ line of an argon laser fiber-coupled to the microscope was used. Emitted fluorescence light was collected with the same objective and then passed through a confocal pinhole and a LP530 long pass emission filter to reach a photomultiplier detector. A stainless-steel chamber Attofluor (Thermo Fisher Scientific) holding the $25 \mathrm{~mm}$ round coverslip was used as a sample cell. A glass coverslip functionalized with pHrodo dye and covered (or not) with a polymer film was mounted in the sample holder, and a droplet of buffer solution with $\mathrm{pH}=9.0$ (ROTI Calipure) was added. For CLSM experiments, the functionalized glass surface was positioned in the middle of the confocal volume (in a vertical direction), and horizontal scans of different regions of the droplet contact line were acquired.

\section{RESULTS AND DISCUSSION}

As an adaptive surface, a random PS/PAA copolymer surface was prepared on $\mathrm{Si}$ wafers by spin-coating. Subsequently, we annealed the samples at $150{ }^{\circ} \mathrm{C}$ for $10 \mathrm{~min}$ in an oven (Figure 1a). The average roughnesses of the films were $0.2 \pm 0.02 \mathrm{~nm}$ on a $500 \times 500 \mathrm{~nm}^{2}$ scale (Figure S1). The thicknesses of the films were $38 \pm 1$ and $67 \pm 1 \mathrm{~nm}$, respectively, as measured by a profilometer (KLA-Tencor Stylus-Profiler model P7). By annealing, we expected the hydrophobic styrene side groups to 


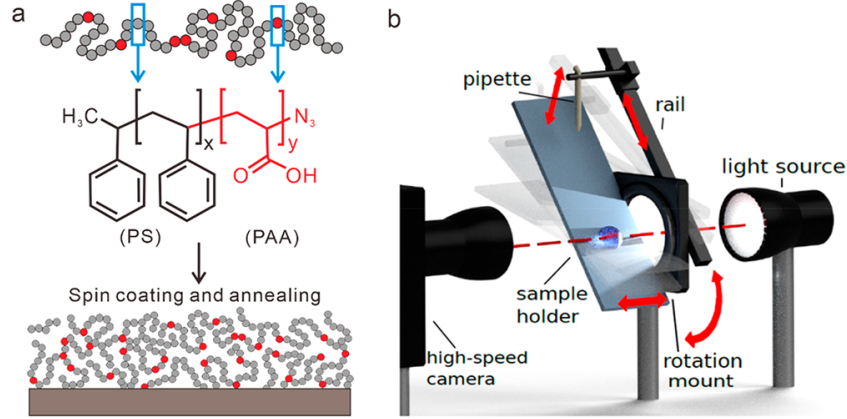

Figure 1. (a) Schematics of the PS/PAA copolymer surface. (b) Schematics of the tilted-plate setup. The corresponding dataprocessing process is explained in the Supporting Information (S4).

enrich at the surface to lower the surface energy. When the PS/ PAA films were exposed to water, they were able to change their wetting properties by swelling and by reorientation of the polymer chain segments at the surface.

We synthesized PS/PAA surfaces with three different styrene/acrylic acid molar ratios: $8.7 / 1,4.2 / 1$, and $2.9 / 1$ (details are in the Methods section). The ratios were revealed by NMR (Figure S2). The glass transition temperature $T_{\mathrm{g}}$ of all three PS/PAA copolymers was measured to be between 100 and $130{ }^{\circ} \mathrm{C}$ (Figure S3).

When measuring the contact angles of films by the traditional sessile drop method, the advancing contact angles of PS and all PS/PAA copolymers were in the range from $95^{\circ}$ to $98^{\circ}$. However, the receding contact angle decreased by $21^{\circ}$ when increasing the acrylic acid content from $8.7 / 1$ to $2.9 / 1$ (Table 1). Compared to pure PS, the receding angle for $2.9 / 1$

Table 1. Dynamic Contact Angles of Copolymer Surfaces with Different PS-to-PAA Ratios ${ }^{a}$

$\begin{array}{cccc}\text { polymer (PS/PAA) } & \theta_{\mathrm{A}}(\mathrm{deg}) & \theta_{\mathrm{R}}(\mathrm{deg}) & \Delta \theta(\mathrm{deg}) \\ 10 / 0 & 97 \pm 1 & 70 \pm 1 & 27 \pm 2 \\ 8.7 / 1 & 98 \pm 1 & 66 \pm 1 & 32 \pm 2 \\ 4.2 / 1 & 96 \pm 1 & 50 \pm 1 & 46 \pm 2 \\ 2.9 / 1 & 95 \pm 1 & 45 \pm 1 & 50 \pm 2\end{array}$

${ }^{a}$ The advancing and receding angles were measured using the sessile drop method.

(PS/PAA) even decreased by $25^{\circ}$. Accordingly, the contact angle hysteresis varies between $27^{\circ}$ and $50^{\circ}$. In this contact angle measurement, the advancing side of the drop always wets a dry surface. In contrast, when measuring the receding contact angles, the surface stayed in contact with water for seconds. Thus, the response of the receding contact angle may indicate that the PS/PAA copolymer surfaces have already started to adapt to water.

The PS/PAA surfaces with a molar ratio of $8.7 / 1$ (PS/PAA) had the highest advancing and receding contact angles. In addition, these films exhibited the lowest contact angle hysteresis $\left(32^{\circ} \pm 2^{\circ}\right)$. According to ${ }^{28-30}$

$$
\sin \alpha_{\mathrm{c}}=\frac{w \gamma_{\mathrm{L}}^{\infty} k}{V \rho g}\left(\cos \Theta_{\mathrm{R}}-\cos \Theta_{\mathrm{A}}\right)
$$

they also show the lowest roll-off angle $\alpha_{\mathrm{c}}$. The roll-off angle is the critical angle of a tilted plate at which a drop of volume $V$ starts to move downward. Here, $w$ is the width of the contact area of the drop, $\rho$ is the density of the liquid, $g=9.81 \mathrm{~m} / \mathrm{s}^{2}$, and $k \approx 1$ is a geometrical factor, which depends on the shape of the drop. ${ }^{31-34}$ Thus, the PS/PAA surface with a molar ratio of $8.7 / 1$ (PS/PAA) allows us to measure the largest velocity range of drops by varying the tilting angle. Therefore, we mainly used an 8.7/1 PS/PAA copolymer surface to measure the velocity-dependent contact angle hysteresis on our tiltedplated setup.

We applied the tilted-plate setup to measure the velocitydependent dynamic contact angles (Figure 1b). The dynamic contact angles were always measured along the same track on the sample. Between measuring subsequent drops, we annealed the sample for $10 \mathrm{~min}$ at $150{ }^{\circ} \mathrm{C}$ to ensure a reproducible dry surface. Before being measured again, the samples were quickly cooled down to room temperature by making a contact to a marble slab for $1 \mathrm{~min}$ (Figures S4 and S5).

We first consider a water drop with a length of $L$ that slides down the PS/PAA surface with a tilted angle $\alpha$ at a velocity $v$ (Figure 2a). During the sliding process, the surface stays in contact with water for $t=L / v$. This contact time can be controlled by adjusting the drop velocity $(v)$, which depends on the tilt angle $(\alpha)$ of the substrate and the distance from the position where the drop was released. The scenarios of both adaptation processes are illustrated at the advancing and receding sides of the drop with a symbolistic view of the orientation of the phenol rings and the carbonic acid groups (Figure 2a). For a sliding drop, we obtain two scenarios for the adaptation of the dynamic contact angle.

(1) When $v \geq l_{\mathrm{SL}} / \tau_{\mathrm{SL}}$, the drop slides too fast to allow adaptation of the surface, and phenol rings remain on the surface. Assuming that this is correct, no change in the contact angle can be measured at higher sliding velocities of the drop. Indeed, for the PS/PAA surface, we measured a constant advancing contact angle of $96^{\circ}$ at the velocity range of $10^{-4}$ up to $0.1 \mathrm{~m} / \mathrm{s}$ (red symbols in Figure $2 \mathrm{~b}$ ). In this velocity range, we did not observe a significant difference to the PS reference sample (blue symbols in Figure $2 \mathrm{~b}$ ). We conclude that annealed PS/PAA films mostly exhibit styrene at the surface.

(2) However, for a drop velocity of $7 \times 10^{-5} \mathrm{~m} / \mathrm{s}$ and below, the advancing contact angle decreased to $87^{\circ}$ for a PS/PAA surface compared to $95^{\circ}$ for a PS surface $\left(v \leq l_{\mathrm{SL}} / \tau_{\mathrm{SL}}\right)$. We interpret this contact angle decrease at very low velocity as an indication of the enrichment of carbonic acid groups at the surface. This enrichment is driven by the contact with water at the advancing side of the sliding drop. It has to take place in a narrow region around the contact line, which influences the contact angle, called the peripheral thickness. Notably, the contact angle decrease at a low sliding velocity does not depend on the thickness of the sample (Figure 2b). Thus, only the surface of the PS/PAA films is involved. The surface can restructure and expose acrylic acid groups or water can penetrate, leading to an increasing dielectric constant of the topmost surface.

To estimate the order of the relaxation time $\tau_{\mathrm{SL}}$ for this process, we fitted eq 1 to the measured advancing angles of the PS/PAA surface. ${ }^{11}$ For the fit, we used $\theta_{\mathrm{a}}^{\infty}=87^{\circ}, \Delta \gamma_{\mathrm{SL}}=0.013$ $\mathrm{N} / \mathrm{m}$, and $\gamma_{\mathrm{L}}^{\infty}=0.072 \mathrm{~N} / \mathrm{m}$ in the velocity range of $5 \times 10^{-6}$ to $0.1 \mathrm{~m} / \mathrm{s}$ (red continuous line in Figure $2 \mathrm{~b}$ ). The fit revealed a ratio of $\frac{l_{\mathrm{SL}}}{\tau_{\mathrm{SL}}}=(5 \pm 2) \times 10^{-5} \mathrm{~m} / \mathrm{s}$. Assuming a peripheral thickness on the order of $l_{\mathrm{SL}}=10 \mathrm{~nm}$ results in a relaxation time $\tau_{\mathrm{SL}}$ of $\sim 0.2 \mathrm{~ms}$. With $l_{\mathrm{SL}}=100 \mathrm{~nm}$, one would estimate $\tau_{\mathrm{SL}}=2 \mathrm{~ms}$. 


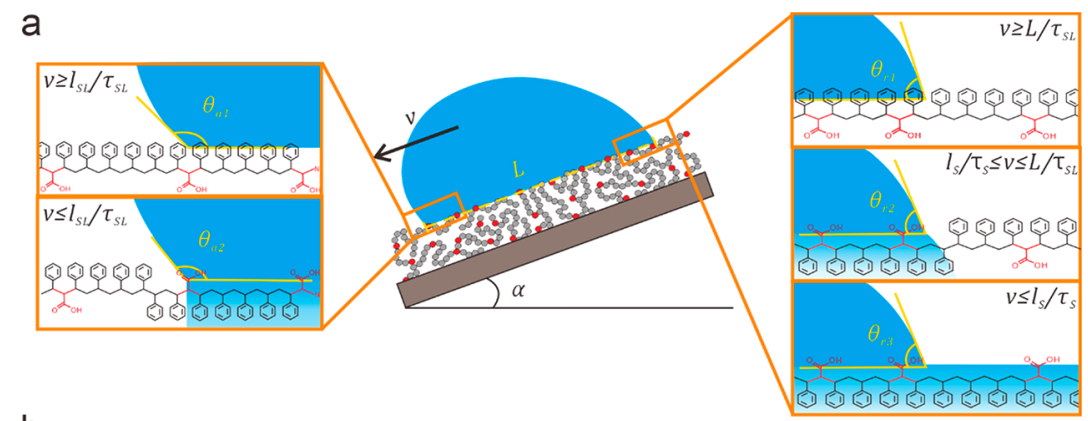

b

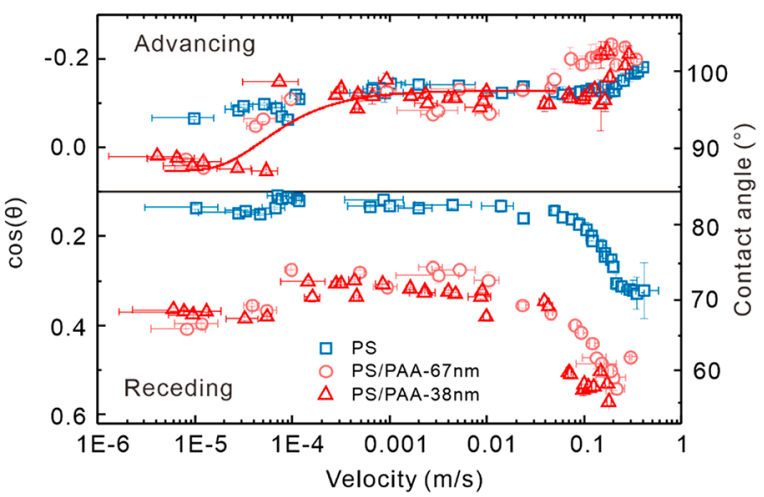

Figure 2. (a) Schematic drawing of the relationship between drop velocity and the change in dynamic contact angles caused by surface adaptation. (b) Contact angles $(\theta)$ and $\cos (\theta)$ vs velocity on the PS and PS/PAA (8.7/1) surfaces with thicknesses of 38 and 67 nm, respectively. Each symbol stands for a measurement of an individual drop. The solid line is according to the adaptation theory. The $R$-square of the fit is 0.87 , in which $R^{2}=1-\frac{\sum_{1}^{n}\left(y_{i}-f_{i}\right)^{2}}{\sum_{1}^{n}\left(y_{i}-\frac{1}{n} \sum_{1}^{n} y_{i}\right)^{2}}\left(y_{i}\right.$, experimental data; $f_{i}$, fitting data).

The presence of acid groups at the surface may be correlated with a diffusion of water into the polymer film. Assuming a diffusion constant $D$ for the PS/PAA film on the order of $10^{-13}$ $\mathrm{m}^{2} / \mathrm{s}$, ${ }^{35}$ we obtained a diffusion depth of $\sim 4-40 \mathrm{~nm}\left(\tau_{\mathrm{d}}=h^{2} /\right.$ $2 D)$. Thus, a likely scenario is that water diffuses into the copolymer, swelling occurs, and the topmost side groups of the copolymer reorient.

Our next step was to demonstrate that the adaptation of the surface to water was also visible at the receding side of the drop. We used a $33 \pm 1 \mu \mathrm{L}$ drop volume in the tilted-plane experiment. This volume resulted in a drop length of $8.1 \mathrm{~mm}$, when the drops slid at a maximum velocity of $0.35 \mathrm{~m} / \mathrm{s}$ in our setup. Thus, the drop stayed in contact with the surface for $\sim 23 \mathrm{~ms}$. This contact time is $\sim 10-100$ times the relaxation time for the PS/PAA surface. Thus, the surface was able to adapt during all the different velocities applied in our measurement setup. The adaptation was reflected in the $\sim 10^{\circ}$ lower receding contact angles of the PS/PAA surface compared to the reference PS surface for all velocities measured (Figure 2b). Thus, contact angle hysteresis was larger for the PS/PAA surface compared to the pure PS surface. Despite the offset in the receding contact angle, the characteristics of velocity-dependent receding contact angles of PS surface and 8.7/1 PS/PAA surface were quite similar (Figure 2b). This indicates that the reverse adaptation time at the receding side of the drop, $\tau_{s}$, was much larger compared to $\tau_{\mathrm{SL}}$. Thus, the topmost side groups of the copolymer did not return to their original positions before contact with water, and it is possible that water stayed partially in the films. We attribute the slight decrease of the receding angles of the PS/ PAA copolymer at a velocity of $\leq 1 \times 10^{-4} \mathrm{~m} / \mathrm{s}$ to the prolonged time that the surface was in contact with water $(\sim 80$ s). For such contact times, the surface became more and more hydrophilic, as we will report later. The increase in advancing angles and decrease in receding angles observed for PS and PS/PSA surfaces at a velocity of $\geq 0.1 \mathrm{~m} / \mathrm{s}$ were both caused by viscous dissipation and the Marangoni effect. ${ }^{36}$

When surfaces adapt to a liquid, the contact angle hysteresis and dynamic contact angles change. Accordingly, the mobility of the drop is affected due to changes in the capillary force,

$$
F_{\text {capillary }}=k w \gamma_{\mathrm{L}}^{\infty}\left(\cos \theta_{\mathrm{R}}-\cos \theta_{\mathrm{A}}\right)
$$

Exemplarily, we calculate the capillary force for a drop volume of $33 \mu \mathrm{L}$, corresponding to a drop width of $5 \mathrm{~mm}$. In comparison to a pure PS surface, the decrease in receding angle on PS/PAA surfaces leads to an increase in capillary force from $\sim 87$ to $\sim 148 \mu \mathrm{N}$ at $0.2 \mathrm{~mm} / \mathrm{s} \leq v \leq 20 \mathrm{~mm} / \mathrm{s}$. At lower velocity, $\leq 20 \mu \mathrm{m} / \mathrm{s}$, where the advancing and receding contact angles adapt, the capillary force increases from $\sim 87$ to $\sim 132$ $\mu \mathrm{N}$. That is, the adaptation of the PS/PAA copolymer surface with a water drop leads to the increase in contact angle hysteresis of $22^{\circ}-25^{\circ}$, corresponding to an increase in capillary force in the range of $45-60 \mu \mathrm{N}$, depending on the sliding velocity.

The receding contact angles decreased with decreasing PSto-PAA ratio (Table 1). We measured the velocity-dependent dynamic contact angles on a PS/PAA surface made by a copolymer with a ratio of $4.2 / 1$ (Figure S6). Then we also fitted eq 1 to the measured advancing angles, leading to $l_{\mathrm{SL}} / \mathrm{SL}$ $=0.04 \mathrm{~m} / \mathrm{s}$. Again, assuming $l_{\mathrm{SL}} \approx 10-100 \mathrm{~nm}$, we obtained a relaxation time of $\tau_{\mathrm{SL}} \approx 0.25-2.5 \mu \mathrm{s}$. Thus, in comparison to the $8.7 / 1 \mathrm{PS} / \mathrm{PAA}$ the $4.2 / 1 \mathrm{PS} / \mathrm{PAA}$ copolymer surfaces, it required less time to adapt to water. For the sliding drop, the magnitude of the advancing contact angle change is similar for 
a

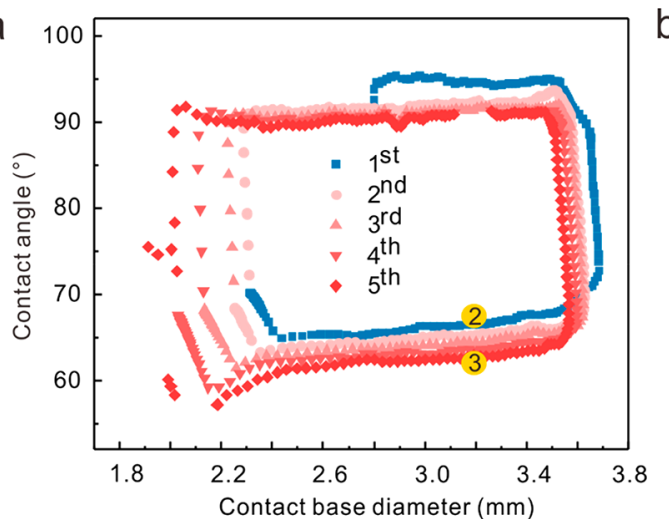

b
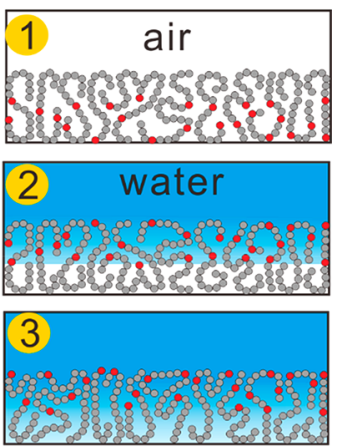

Figure 3. (a) Contact angle vs contact base diameter on the $67 \mathrm{~nm}$ PS/PAA (8.7/1) surface. The cycle's orders of repeated inflating and deflating processes are represented by $1 \mathrm{st}-5 \mathrm{th}$. The 5 repeated tests required $4 \pm 0.5 \mathrm{~min}$, corresponding to $50 \pm 1 \mathrm{~s}$ for each cycle. (b) Possible arrangement of the PS/PAA copolymer at the interface corresponding to positions 2 and 3 in (a). In particular, state 1 in air is the original state of the copolymer before a drop was deposited on the surface.

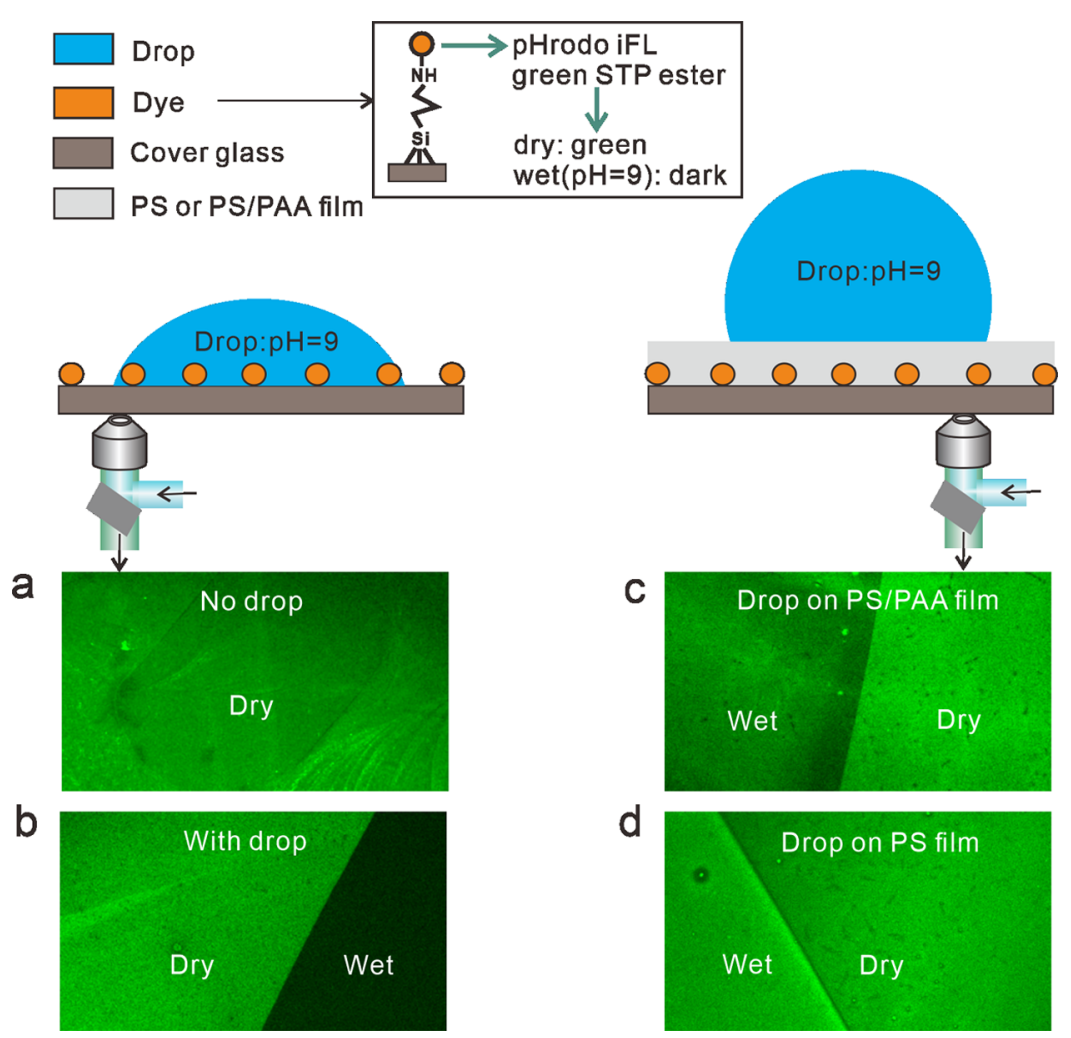

Figure 4. Schematic drawing of water penetration into the copolymer film using confocal microscopy. The images show fluorescence intensity maps (a) before a drop $(\mathrm{pH}=9)$ was deposited on a glass surface grafted with a $\mathrm{pH}$-sensitive dye, (b) after a drop $(\mathrm{pH}=9)$ was deposited on the dye-grafted glass surface, $(\mathrm{c})$ after a drop $(\mathrm{pH}=9)$ was deposited on a 8.7/1 PS/PAA film spin-coated above the dye-grafted glass surface, and (d) after a drop $(\mathrm{pH}=9)$ was deposited on a PS film spin-coated above the dye-grafted glass surface. The detected area for (b), (c), and (d) was a three-phase area.

the surfaces made from $8.7 / 1$ and 4.2/1 PS/PAA copolymers. Thus, we conclude that water diffuses faster into the copolymer that has more acrylic acid content. For the receding side, the 4.2/1 PS/PAA surface has also already completely adapted to the water. We measured a receding angle of $55^{\circ} \pm$ $2^{\circ}$, which is almost $15^{\circ}$ lower than that of the $8.7 / 1$ PS/PAA surface (Figure S6).

To study the adaptation upon contact with water over a longer time scale, we measured the advancing and receding contact angles of PS/PAA films by repeatedly inflating and deflating a water drop. The flow rate used during the measurement was $0.5 \mu \mathrm{L} / \mathrm{s}$ (this is the lowest flow rate that our instrument could achieve), corresponding to a contact line velocity of $(8 \pm 1) \times 10^{-5} \mathrm{~m} / \mathrm{s}$ (beginning with small drop volumes) to $(6 \pm 1) \times 10^{-5} \mathrm{~m} / \mathrm{s}$ (ending with the maximum inflated drop volume). The first contact angle measurement was performed on a pristine PS/PAA surface, as used in the sliding drop experiments (blue data points in Figure 3a). In the first cycle of the contact angle measurement during inflation, the advancing angle was $95^{\circ} \pm 1^{\circ}$. The receding contact angle measured during deflation was $66^{\circ} \pm 1^{\circ}$. This contact angle slightly decreased during deflation of the drop. We inflated the 
drop again (red, round symbols in Figure 3a). In the second cycle, the advancing contact angle decreased to $92^{\circ} \pm 1^{\circ}$. The corresponding receding contact angle decreased to $64^{\circ} \pm 1^{\circ}$. Subsequently, the drop was inflated again three more times. In the 3rd-5th cycles, the advancing contact angle only slightly decreased to $90^{\circ} \pm 1^{\circ}$. The corresponding receding contact angles kept decreasing continuously for each cycle by $\sim 1^{\circ}$. At the fifth cycle, a receding contact angle of $62^{\circ} \pm 1^{\circ}$ was measured (Figure 3a).

We attributed the decrease in advancing and receding contact angles to water penetrating into the PS/PAA film and staying in the film for the subsequent cycles. Thus, in the 2nd5th cycles, the surface appears more hydrophilic. In addition, the presence of water in the film allows the hydrophilic PAA segment to move to the interface (Figure $3 b$, states 2 and 3 ). Therefore, the advancing angles and receding angles gradually decreased after every cycle.

It is worth mentioning that during successive inflations of the drop the advancing contact angle never reached the value of the pristine sample. Thus, $\tau_{\mathrm{s}}$ is longer than the time for one measurement cycle, which is $50 \pm 5 \mathrm{~s}$. For comparison, we used pure PS surfaces as a reference. Both the advancing and receding angles on the PS surfaces remained almost constant $\left(\theta_{\mathrm{A}}=97^{\circ}, \theta_{\mathrm{R}}=73^{\circ}\right)$ even after five inflation/deflation cycles (Figure S7). The small differences in the contact angles measured by sliding drop and sessile drop for the same samples at their original state and final state can be caused by the different measuring methods and fitting models. We used a polynomial model for sliding drop and an elliptical model for sessile drop experiments.

To determine whether water molecules are able to diffuse in the PS/PAA film and if they can reach the $\mathrm{SiO}_{2}$ substrate interface, we grafted a $\mathrm{pH}$-sensitive fluorophore ( $\mathrm{pHrodo} \mathrm{iFL}$ STP eater, ThermoFisher) to the surface of a glass coverslip. This fluorophore emits green light with an emission maximum at a wavelength of $525 \mathrm{~nm}$ after excitation by a $488 \mathrm{~nm}$ laser. Fluorescence decreases when it comes into contact with basic water $(\mathrm{pH}=9)$. To confirm the activity of the $\mathrm{pH}$-sensitive dye, we deposited a drop with $\mathrm{pH}=9$ on the surface of a dyegrafted glass surface and recorded the fluorescence intensity using a confocal microscope. The fluorescence intensity of the dry area is higher than that in the wet area (Figure $4 a$ and $b$ ).

We used this fluorophore to verify the presence of water at the PS/PAA/substrate interface. We spin-coated the PS/PAA polymer film (thickness $=45 \mathrm{~nm}$ ) on a dye-grafted glass surface and added a drop $(\mathrm{pH}=9)$ onto the polymer surface. The fluorescence image recorded $\sim 1 \mathrm{~min}$ after adding the drop showed a slight decrease in fluorescence (Figure 4c). Thus, we concluded that water had penetrated through the polymer film and reached the dye layer grafted on the glass surface. In comparison, the pure PS film showed no decrease in fluorescence (Figure 4d). The latter rules out optical artifacts due to the presence of a water drop on top of the surface, which could also lead to changes in the intensity of the backscattered light.

\section{CONCLUSION}

The surface of a PS/PAA copolymer film adapts upon contact with water. Most likely, water penetrates in the uppermost layer and acrylic acid groups become exposed to the water interface. On the basis of a recently proposed theory, we estimated the time scale of the swelling and reorganization process to be $\ll 1 \mathrm{~s}$. For a $8.7 / 1 \mathrm{PS} / \mathrm{PAA}$ surface and for sliding velocities of drops $\geq 7 \times 10^{-4} \mathrm{~m} / \mathrm{s}$, the advancing contact line "sees" a styrene-dominated surface. For a sliding velocity $\leq 7 \times$ $10^{-4} \mathrm{~m} / \mathrm{s}$, the acrylic acid groups have sufficient time to get exposed so that the advancing contact line of the drop can be influenced by the acrylic acid groups. This adaptation gradually leads to the decrease of advancing angle. In the case of a 4.2/1 PS/PAA surface, the critical sliding velocity increases to $3 \times$ $10^{-2} \mathrm{~m} / \mathrm{s}$. In both cases, at the receding contact line, the surface has adapted and more acid groups are exposed. In particular, the adaptation time scale could depend on phase separation of PAA and PS moieties. The latter would be even more pronounced for block copolymer morphologies. The adaptation processes of the surface influence the mobility of drops by increasing the capillary force in the range of 45-60 $\mu \mathrm{N}$ in comparison to a pure PS reference surface.

\section{ASSOCIATED CONTENT}

\section{sI Supporting Information}

The Supporting Information is available free of charge at https://pubs.acs.org/doi/10.1021/acs.langmuir.0c03226.

Surface morphology characterization by scanning force microscopy; copolymer composition characterization by NMR; $T_{g}$ of PS, PAA, and PS/PAA copolymers; data processing of sliding drops; evidence of drying "wet" surfaces by annealing; adaptation on $4.2 / 1$ PS/PAA surface by sliding drops; and dynamic contact angles of PS surface measured by sessile drop (PDF)

\section{AUTHOR INFORMATION}

\section{Corresponding Author}

Rüdiger Berger - Max Planck Institute for Polymer Research, 55128 Mainz, Germany; 이이.org/0000-0002-40840675; Email: berger@mpip-mainz.mpg.de

\section{Authors}

Xiaomei Li - Max Planck Institute for Polymer Research, 55128 Mainz, Germany

Simon Silge - Max Planck Institute for Polymer Research, 55128 Mainz, Germany

Alexander Saal - Max Planck Institute for Polymer Research, 55128 Mainz, Germany

Gunnar Kircher - Max Planck Institute for Polymer Research, 55128 Mainz, Germany

Kaloian Koynov - Max Planck Institute for Polymer Research, 55128 Mainz, Germany; 이이.org/0000-0002-40628834

Hans-Jürgen Butt - Max Planck Institute for Polymer Research, 55128 Mainz, Germany; 이이.org/00000001-5391-2618

Complete contact information is available at:

https://pubs.acs.org/10.1021/acs.langmuir.0c03226

\section{Notes}

The authors declare no competing financial interest.

\section{ACKNOWLEDGMENTS}

Our thanks go to Jürgen Thiel for synthesizing the PS polymer and to Uwe Rietzler and Helma Burg for their technical support. We acknowledge partial funding by the Deutsche Forschungsgemeinschaft (DFG, German Research Foundation), Project-ID 265191195-SFB 1194, and within the German Priority Program 2171. 


\section{REFERENCES}

(1) Lavielle, L.; Schultz, J. Surface properties of graft polyethylene in contact with water: I. Orientation phenomena. J. Colloid Interface Sci. 1985, 106 (2), 438-445.

(2) de Crevoisier, G.; Fabre, P.; Corpart, J.-M.; Leibler, L. Switchable tackiness and wettability of a liquid crystalline polymer. Science 1999, 285 (5431), 1246-1249.

(3) Grundke, K.; Pöschel, K.; Synytska, A.; Frenzel, R.; Drechsler, A.; Nitschke, M.; Cordeiro, A. L.; Uhlmann, P.; Welzel, P. B. Experimental studies of contact angle hysteresis phenomena on polymer surfaces-Toward the understanding and control of wettability for different applications. Adv. Colloid Interface Sci. 2015, 222, 350-376.

(4) Sui, X.; Zapotoczny, S.; Benetti, E. M.; Memesa, M.; Hempenius, A. M.; Vancso, G. J. Grafting mixed responsive brushes of poly $(\mathrm{N}$ isopropylacrylamide) and poly(methacrylic acid) from gold by selective initiation. Polym. Chem. 2011, 2, 879-884.

(5) Liu, C.; Lopes, M. C.; Pihan, S. A.; Fell, D.; Sokuler, M.; Butt, H.-J.; Auernhammer, G. K.; Bonaccurso, E. Water diffusion in polymer nano-films measured with microcantilevers. Sens. Actuators, $B$ 2011, 160 (1), 32-38.

(6) Yoon, J.; Cai, S.; Suo, Z.; Hayward, R. C. Poroelastic swelling kinetics of thin hydrogel layers: comparison of theory and experiment. Soft Matter 2010, 6 (23), 6004-6012.

(7) Zhang, H.; Gao, X.; Chen, K.; Li, H.; Peng, L. Thermo-sensitive and swelling properties of cellouronic acid sodium/poly (acrylamideco-diallyldimethylammonium chloride) semi-IPN. Carbohydr. Polym. 2018, 181, 450-459.

(8) Ionov, L.; Minko, S. Mixed polymer brushes with locking switching. ACS Appl. Mater. Interfaces 2012, 4 (1), 483-489.

(9) Ochsmann, J. W.; Lenz, S.; Lellig, P.; Emmerling, S. G. J.; Golriz, A. A.; Reichert, P.; You, J.; Perlich, J.; Roth, S. V.; Berger, R.; Gutmann, J. S. Stress-Structure Correlation in PS-PMMA Mixed Polymer Brushes. Macromolecules 2012, 45 (7), 3129-3136.

(10) Lee, S.; Flores, S. M.; Berger, R.; Gutmann, J. S.; Brehmer, M.; Conrad, L.; Funk, L.; Theato, P.; Yoon, D. Y. Sheeting, Temperature dependence of surface reorganization characteristics of amphiphilic block copolymer in air and in water studied by scanning force microscopy. J. Plast. Film Sheeting 2015, 31 (4), 434-448.

(11) Butt, H. J.; Berger, R.; Steffen, W.; Vollmer, D.; Weber, S. A. L. Adaptive Wetting-Adaptation in Wetting. Langmuir 2018, 34 (38), 11292-11304.

(12) Dupont-Gillain, C. C.; Adriaensen, Y.; Derclaye, S.; Rouxhet, P. G. Plasma-oxidized polystyrene: wetting properties and surface reconstruction. Langmuir 2000, 16 (21), 8194-8200.

(13) Vaidya, A.; Chaudhury, M. K. Synthesis and surface properties of environmentally responsive segmented polyurethanes. J. Colloid Interface Sci. 2002, 249 (1), 235-45.

(14) Honda, K.; Morita, M.; Sakata, O.; Sasaki, S.; Takahara, A. Effect of Surface Molecular Aggregation State and Surface Molecular Motion on Wetting Behavior of Water on Poly(fluoroalkyl methacrylate) Thin Films. Macromolecules 2010, 43 (1), 454-460.

(15) Honda, K.; Yamamoto, I.; Morita, M.; Yamaguchi, H.; Arita, H.; Ishige, R.; Higaki, Y.; Takahara, A. Effect of $\alpha$-substituents on molecular motion and wetting behaviors of poly(fluoroalkyl acrylate) thin films with short fluoroalkyl side chains. Polymer 2014, 55 (24), 6303-6308.

(16) Zhao, J.; Wang, M.; Gleason, K. K. Stabilizing the Wettability of Initiated Chemical Vapor Deposited (iCVD) Polydivinylbenzene Thin Films by Thermal Annealing. Adv. Mater. Interfaces 2017, 4 (18), 1700270.

(17) Neto, C.; James, M.; Telford, A. M. On the Composition of the top layer of microphase separated thin PS-PEO films. Macromolecules 2009, 42, 4801-4808.

(18) Inutsuka, M.; Tanoue, H.; Yamada, N. L.; Ito, K.; Yokoyama, $\mathrm{H}$. Dynamic contact angle on a reconstructive polymer surface by segregation. RSC $A d v .2017$, 7, 17202.
(19) Crowe, J. A.; Genzer, J. Creating responsive surfaces with tailored wettability switching kinetics and reconstruction reversibility. J. Am. Chem. Soc. 2005, 127 (50), 17610-17611.

(20) Farris, S.; Introzzi, L.; Biagioni, P.; Holz, T.; Schiraldi, A.; Piergiovanni, L. Wetting of biopolymer coatings: contact angle kinetics and image analysis investigation. Langmuir 2011, 27 (12), $7563-74$.

(21) Holly, F. J.; Refojo, M. F. Wettability of hydrogels I. Poly (2hydroxyethyl methacrylate). J. Biomed. Mater. Res. 1975, 9 (3), 315326.

(22) Yasuda, H.; Sharma, A. K.; Yasuda, T. Effect of orientation and mobility of polymer molecules at surfaces on contact angle and its hysteresis. J. Polym. Sci., Polym. Phys. Ed. 1981, 19 (9), 1285-1291.

(23) Lee, S. H.; Ruckenstein, E. Surface restructuring of polymers. J. Colloid Interface Sci. 1987, 120 (2), 529-536.

(24) Wong, W. S. Y.; Hauer, L.; Naga, A.; Kaltbeitzel, A.; Baumli, P.; Berger, R.; D'Acunzi, M.; Vollmer, D.; Butt, H. J. Langmuir 2020, 36 (26), 7236-7245.

(25) Bartell, F. E.; Bjorklund, C. W. Hysteresis of contact angles. J. Phys. Chem. 1952, 56 (4), 453-457.

(26) Ruckenstein, E.; Gourisankar, S. V. Surface restructuring of polymeric solids and its effect on the stability of the polymer-water interface. J. Colloid Interface Sci. 1986, 109 (2), 557-566.

(27) Sedev, R. V.; Budziak, C. J.; Petrov, J. G.; Neumann, A. W. Dynamic contact angles at low velocities. J. Colloid Interface Sci. 1993, 159 (2), 392-399.

(28) Furmidge, C. G. L. Studies at phase interfaces. I. The sliding of liquid drops on solid surfaces and a theory for spray retention. J. Colloid Sci. 1962, 17 (4), 309-324.

(29) Olsen, D. A.; Joyner, P. A.; Olson, M. D. The sliding of liquid drops on solid surfaces. J. Phys. Chem. 1962, 66 (5), 883-886.

(30) Wolfram, E. Liquid drops on a tilted plate, contact angle hysteresis and the Young contact angle. Wetting, Spreading Adhes. 1978, 213-222.

(31) Dussan V, E. B. On the ability of drops or bubbles to stick to non-horizontal surfaces of solids. Part 2. Small drops or bubbles having contact angles of arbitrary size. J. Fluid Mech. 1985, 151, 1-20.

(32) Extrand, C. W.; Gent, A. N. Retention of liquid drops by solid surfaces. J. Colloid Interface Sci. 1990, 138 (2), 431-442.

(33) ElSherbini, A. I.; Jacobi, A. M. Retention forces and contact angles for critical liquid drops on non-horizontal surfaces. J. Colloid Interface Sci. 2006, 299 (2), 841-849.

(34) Antonini, C.; Carmona, F. J.; Pierce, E.; Marengo, M.; Amirfazli, A. General methodology for evaluating the adhesion force of drops and bubbles on solid surfaces. Langmuir 2009, 25 (11), 6143-6154.

(35) Fike, L. R. Transport properties of polystyrene above and below the glass transition temperature; Texas Tech University: 1983.

(36) Henrich, F.; Fell, D.; Truszkowska, D.; Weirich, M.; Anyfantakis, M.; Nguyen, T.-H.; Wagner, M.; Auernhammer, G. K.; Butt, H.-J. Influence of surfactants in forced dynamic dewetting. Soft Matter 2016, 12 (37), 7782-7791. 